\title{
SUMMARIES OF TECHNICAL PAPERS RECEIVED
}

\author{
(PREPARED BY THE EDITOR)
}

\section{Aerodynamics}

Preliminary Investigation on Boundary Layer Control by means of Suction and Pressure with the U.S.A. 27 Airfoil. F. G. Reid and M. J. Bamber. N.A.C.A. Technical Note No. 286. Washington, May, 1928.

\section{SUMmary.}

Tests were made on a U.S.A.27 airfoil with various slot shapes and combinations, and at various amounts of pressure or suction on the slots.

The lift of airfoils can be increased by removing or by accelerating the boundary layer.

Removing the boundary layer by suction is more economical than to accelerate it by jet action. Gauze-covered suction slots apparently give the best results. When not in operation, all suction slots tested had a detrimental effect upon the aerodynamic characteristics of the airfoil which was not apparent with the backward opening pressure slots.

Thick blunt-nosed airfoils would seem to give the best results with boundary layer control.

The Connection between Lift and Circulation for an Inclined Flat Plate. A. Fage, A.R.C.Sc., and F. C. Johansen, B.Sc. R. \& M. No. ri 39 (Ae. 3o8). H.M.S.O., January, 1928.

\section{SUMmaRY.}

It is shown that the Kutta-Joukowski relationship between lift and circulation holds very closely for an inclined flat plate in an air stream, provided that the contour around which the circulation is taken is large and that it cuts the wake in a straight line perpendicular to the direction of the undisturbed motion. Also the total strength of the vorticity at the front of the plate can be determined from observations of pressure on the plate.

The Structure of Vortex Sheets. A. Fage, A.R.C.Sc., and F. C. Johansen, B.Sc. R. \& M. No. II 43 (Ae. 3II). H.M.S.O., August, 1927.

\section{Summary.}

The structure of the sheets shed from infinitely long bodies of widely different cross section has been examined. Measurements have also been made to determine the principal characteristics of the vortex sheets formed at some distance behind each body.

It is established from estimations of vorticity and also from observations of total head, that the outer boundary of a sheet can be taken as a line drawn through the points in the field of maximum velocity $\left(V_{1}\right)$, and the inner boundary as the line passing through the points of minimum velocity $\left(V_{2}\right)$. The motion in a sheet is steady near the body, except possibly near the inner boundary.

The pressure at points in an outer boundary is slightly greater than that in the dead air region behind the body.

Air flows into a sheet through both boundaries, but at a greater rate through the outer boundary. The simple expression $\left(V_{1}^{2}-V_{2}^{2}\right) / 2 V_{0}^{2}$ gives a close estimate of the total amount of vorticity passing a section of a sheet in unit time. 

rate.

Vorticity is shed from the two sides of an asymmetric body at the same

The variation of velocity across a section of a sheet, and also the rate of increase of the breadth with the distance downstream, depend on the form of the body. The velocity distribution in the sheet shed from a flat plate normal to the stream is in close agreement with that predicted by the Prandtl-Tollmien theory.

At constant forward speed, both the frequency and the longitudinal spacing of the individual vortices in the vortex street depend largely on the lateral spacing between the sheets, and to a smaller extent on the form of the body.

On the Flow of a Compressible Fluid Past an Obstacle. Dr. H. Lamb, F.R.S. R. \& M. No. iा 56 (Ae. 32 I). H.M.S.O., April, 1928.

\section{SUMmary.}

An extension of the flow past a cylinder without circulation of the fluid round it, discussed originally by Rayleigh, to include circulation.

\section{Aerofoils}

Wind Tunnel Tests on Aerofoil R.A.F. $3^{6}$. F. B. Bradfield and K. W. Clark, B.Sc., D.I.C. R. \& M. No. Ir 47 (Ae. 315), December, I927.

\section{Summary.}

Lift, drag and centre of pressure have been determined at $R=0.25^{1} \times 10^{6}$ and $0.33^{6} \times 10^{6}$, and minimum drag was measured from $R=0.168 \times 10^{6}$ to $R=1.00 \times 10^{6}$. The main characteristics at $R=0.251 \times 10^{6}$ are given, compared with other aerofoils in the series.

Scale Effect on Three Aerofoils at Low Values of LV, R.A.F. 32, Göttingen 433 and Göttingen 4ro, with 2 per cent. Centre Line Camber. F. B. Bradfield. R. \& M. No. III7 (Ae. 290). H.M.S.O., August, 1927.

\section{Summary.}

Lift and drag were measured down to $L V=2 \frac{1}{2}$. Minimum drag at $L V=2 \frac{1}{2}$ is about 0.025 for R.A.F. 32 section or for Göttingen 410 (cambered), and about 0.04 for Göttingen 43.3. As the scale decreases the no-lift angle occurs at a more and more positive incidence, the slope of the lift curve remaining roughly constant. In the case of Göttingen 4.3.3 and Göttingen 410 (cambered), the ordinary stall has disappeared from the lift curves at the lowest $L V$ 's tested, the lift decreasing gradually over a $40^{\circ}$ incidence range.

Aerodynamic Characteristics of Aerofoils-V. N.A.C.A. Report No. 286. Washington, I928.

\section{SUMMARY.}

Continuation of Reports Nos. 93, 124, 182 and 244.

This collection of data on aerofoils has been made from the published reports of a number of the leading aerodynamical laboratories of the United States and Europe, and is presented in a uniform series of charts and tables suitable for the use of designing engineers and for general reference.

Wind Tunnel Tests on a R.A.F. I5 Aerofoil with Pilot Planes. F. B. Bradfield and K. W. Clark, B.Sc., D.I.C. R. \& M. No. II45 (Ae. 313). October, 1927. H.M.S.O.

\section{Summary.}

A pilot plane is an auxiliary aerofoil pivoted ahead of a wing so as to provide automatically a slotted wing at high incidence without much increase of drag at fine angles. The maximum lift region was investigated with pilot planes of 
chord $7 \frac{1}{2}$ per cent., to per cent. and I $2 \frac{1}{2}$ per cent. of the main chord, trying different hinge positions relative to the main wing in each case. The drag of the best lift arrangement for each pilot plane was measured, and the angle at which the pilot plane floated freely was recorded.

With a so per cent. pilot plane, the maximum lift coefficient of a R.A.F. I 5 aerofoil may be increased to 0.80 , and the increase of drag at top speed is less than the minimum drag of the pilot plane when tested alone; but the increase of drag is greater on the climb. By making the stop which limits the pilot plane angle vary with the aileron angle, the pilot plane gives an efficient slot-and-aileron control, the pilot plane reversing the sign of the yawing moments at angles just over the stall. With fixed stop the aileron control is much improved by fitting pilot planes, but the effective control may still be poor owing to the pilot plane increasing the stability.

\section{Aeronautics-General}

Flying. Lieut.-Colonel W. Lockwood Marsh, M.A. May, 1928.

\section{Summary.}

One of a series in the modern pictorial library. A short general account, extensively illustrated, of aeroplanes, seaplanes and airships.

\section{Aeroplanes-General}

Elements of Aviation. 'V. E. Clark. Ronald Press Company. ${ }_{1928 .}$

\section{Chapter Headings.}

The Air and the Airfoil; Airfoils and Airflow; Effects of Airflow; Stability and Control; Propeller Effects; Accelerated Flight; The Earth's Atmosphere and the Effects of Altitude; Parts of an Airplane; Weights and Dimensions.

\section{Aeroplanes-Full Scale Experiments}

Full-Scale and Model Measurements of Lift and Drag of a Bristol Fighter fitted with R.A.F. 34 Wings. J. K. Hardy, B.A., and A. S. Hartshorn, B.Sc. R. \& M. No. II46 (Ae. 314). November, 1927. H.M.S.O.

\section{Summary.}

The report forms one of a series describing comparisons between the fullscale and model lift and drag of biplanes fitted with thick wings. The lift and drag of a Bristol Fighter with wings of R.A.F. 34 section have been determined for the full-scale aeroplane and for a $1 /$ Ioth scale at speeds of 40 , 60 and $80 \mathrm{ft}$. $/ \mathrm{sec}$.

The maximum lift coefficient $\left(0.5^{6}\right)$ is higher for the full-scale aeroplane than for the model $(0.52)$, but the increase is not so great as for R.A.F. $3^{\circ}$ or R.A.F. 31. The minimum drag coefficients, both model and full-scale, are the same as for R.A.F. $3^{1}$, which has the same centre line camber and thickness, but is not reflexed.

\section{Aeroplanes-Performance}

Notes on Performance Testing. H. L. Stevens, B.A., and A. E. Woodward Nutt, B.A. R. \& M. No. Ir 40 (Ae. 309). February, I928. H.M.S.O.

\section{SUMmary.}

Describes methods of performance testing and analysis at present in use at Martlesham Heath. The straightforward method of direct measurement of climbs and speeds and the research method of Mr. Capon are discussed. The present method is a combination of both. 


\section{Airships}

The Resistance of the International Airship Models Measured in the Wind Tunnel of the Royal College of Science, South Kensington, S.W.7. Professor F. T. Hill and G. Tanner, A.C.G.I., D.I.C. H.M.S.O., March; 1928.

\section{Summary.}

Tables of results are given. These are the models already tested in the wind tunnels in America, Japan, Italy, France, the R.A.E. and the N.P.L.

\section{Airscrews}

Tests of a Metal Airscrew in a Closed Tunnel for Comparison with American Tests in an Open Jet Tunnel. H. C. H. Townend, B.Sc., and J. H. Warsop. R. \& M. No. I137 (Ae. 307). H.M.S.O., December, 1927.

Summary.

Results of tests are shown in figures and tables. Tests were carried out in $7 \mathrm{ft}$. closed tunnel. Comparison with the American results shows excellent agreement on thrust over the entire range, while the torque is slightly higher, so that the maximum efficiency is about $1 \frac{1}{2}$ per cent. lower than that obtained in the American test. The screw tested was a $3^{\mathrm{ft}}$. diameter two-bladed metal airscrew of medium pitch.

\section{Autogyro .}

Further Development of Autogyro Theory. Parts I. and II. C. N. H. Lock, M.A. R. \& M. No. I, I27 (Ae. 209). March, 1927. H.M.S.O.

\section{Stumary.}

The object of the present $R$. \& M. is to develop the theory of the autogyro and remove some of the approximations of $R$. \& M. No. IIII.

After working out the force components, an alternative method of determining the drag is developed, based on considerations of energy loss; it has been verified that the resulting formulæ give results identical with those already obtained, and, being more simple, take the place of the rather complicated formulæ for the longitudinal force.

The ratio of the value of maximum lift/drag of the present investigations (the true value for heavy blades subject to the assumptions that the coefficient of axial velocity through the disc is constant over the disc and is a small quantity; and the lift coefficient of a blade element is proportional to the incidence, and the profile drag coefficient is constant) to the values given in R. \& M. No. II I is as follows:-

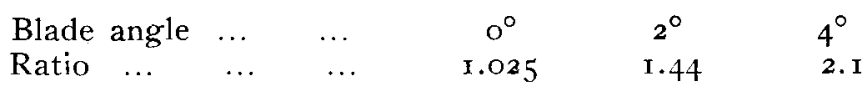

On account of the errors introduced by the assumptions the actual value of lift/drag is probably lower than obtained here, and may in fact be closer to the value obtained in R. \& M. No. I III.

\section{Bombs and Ballistics}

The Experimental Determination of the Trajectory of Aircraft Bombs. H. E. Wimperis, C.B.E., M.A. R. \& M. No. I,121 (Ae. 294). June, 1928. H.M.S.O.

\section{Summary.}

Full scale experiments were made at various air stations and use was made of a coal mine shaft in Yorkshire. It was found that the particle trajectory was sufficiently closely followed for the practical purposes of the moment, and a tech- 
nique of bomb sighting was built thereon. This led to the introduction into the Air Forces of Great Britain of the system of sighting described. A full scale study was also made of the under-water trajectory of a bomb above the sea; this was required for the purposes of the anti-submarine aircraft patrol.

\section{Dopes}

Researches on Cellulose Acetate and its Solution. Composition of Cellulose Acetate Lacquer for Aeroplane Dope. K. Atsuki, and R. Shinoda, Y. Tanaka.

II. Stability of Cellulose Acetate.

III. Stabiliser for Cellulose Acetate.

IV. On the Acetylation of Cellulose.

V. Relation of Temperature and Time of Ripening to the Viscosity of Cellulose Acetate.

Reports of the A.R.I., Tokyo Imperial University, Nos. 32-36. FebruaryMarch, 1928.

\section{Stmmaries.}

I. The optimum composition of the mixed solvent for cellulose acetate in the preparation of an aeroplane dope was studied by determining the solvent power of the mixed solvent, the viscosity of the cellulose acetate solution in the mixed solvent, and the tensile strength and elongation of the film produced. It is proved that the composition of the mixed solvent, which has the highest solvent power and gives the lowest viscosity of cellulose acetate solution, produces a film of the highest tensile strength and elongation.

Using the most common solvents, the optimum composition of the mixed solvent is acetone 60 per cent., ethyl alcohol $15^{-1} 7$ per cent., benzene 19-21 per cent, benzyl alcohol $\mathrm{I}-2$ per cent., and triphenyl phosphate $1-2$ per cent., and the optimum concentration of cellulose acetate is $7-9$ per cent.

II. The spontaneous decomposition of cellulose acetate, prepared by using sulphuric acid as a catalyst, is governed by the sulphuric acid, which is present as a free state or as an adsorption compound or as the ester of cellulose. The amount of the sulphuric acid retained decreases as the ripening of cellulose acetate proceeds, because of the saponification of the sulphuric ester, and because of the coagulating power becoming weak, making the diffusion of acid easy. The decomposition products of cellulose by oxidation and hydrolysis have no direct concern with the stability.

III. Calcium naphthenate is found to be the most effective stabiliser for cellulose acetate as well as for cellulose nitrate. The amount of calcium naphthenate to be added must be calculated from the amount of the sulphuric acid present, but in general cases $I-2$ per cent. on cellulose acetate will be sufficient.

IV. In an excessive acetylation, the cellulose transforms successively as peptisation-acetylation-acetolysis-equilibrium. Cellibiose and glucose, the end products of the acetolysis, are not formed at the beginning of the acetylation. The reactions involved in the acetylation are not clearly known. The authors have carried out experiments for the object of studying the modification and acetylation of cellulose in general.

V. The viscosity may be used as the indicator for measuring the degree of the ripening of cellulose acetate, and it is governed by the temperature and time of the ripening, if the other conditions are kept constant. The relation between the temperature and time of the ripening to produce a cellulose acetate of a given viscosity was experimented upon, and from the result of the experiment as well as from the theoretical calculation, the expression $T=a e^{k z}$ was obtained for a given viscosity, in which $T$ is the temperature and $z$ is the time of ripening, and $a$ and $k$ are contents. 


\section{Engines}

Motoring Losses in Internal Combustion Engines. H. Moss, D.Sc., A.R.C.S., D.I.C. R. \& M. No. 1128 (E. 27). H.M.S.O., July, 1927.

\section{Summary.}

The total frictional losses of a Benz single cylinder engine were found when motored at speeds of 1,200 r.p.m. and 900 r.p.m. with air densities ranging from normal to twice normal. The pumping losses, measured by an R.A.E. electrical indicator, were deducted, the remainder being the sliding friction of engine and accessories.

The sliding friction under power may be greater than when motoring by the equivalent of $3.5 \mathrm{lbs}$. per sq. in. M.E.P. due to this cause. With an increase of 3.5 lbs./sq. in. M.E.P., found by Ricardo due to carbonisation, the magnitude of the effect when running under power with a normal spark setting is fully explained.

The Determination of the Horse-Power Height Factor of Engines from the Results of Type Trials of Aircraft. J. D. Coales and A. L. Lingard. R. \& M. No. II $4^{1}$ (Ae. 310). H.M.S.O., October, I927.

\section{Summary.}

The average values of the power factor have been determined for heights in a standard atmosphere from the type trial reports of a large number of aircraft. The method of obtaining the power factors of an engine in a standard atmosphere appears to yield reasonable values for this factor; but it gives no clue as to the dependence of the factor upon pressure or density respectively. This is because temperature, pressure and density in a standard atmosphere can each be expressed as definite functions of either one or both of the others.

\section{Engines-Supercharging}

A Preliminary Investigation of Supercharging an Air-Cooled Engine in Flight. Marsden Ware and Oscar W. Schey. N.A.C.A. Report No. 283. Washington, 1928 .

\section{SUmmary.}

Service training aeroplanes were used, equipped with production types of Wright engines. An N.A.C.A. Roots' type supercharger was driven from the rear of the engine. In addition to measuring those quantities that would enable the determination of the climb performance, measurements were made of the cylinder head temperatures and the carburettor pressures and temperatures. The supercharging equipment was not removed from the aeroplane when making flights without supercharging, but a by-pass valve, which controlled the amount of supercharging by returning to the atmosphere the surplus air delivered by the supercharger, was left full open.

With the supercharger so geared that the ground level pressure could be maintained to 18,500 feet, it was found that the absolute ceiling was increased from 19,400 to $3^{2,000}$ feet, that the time to climb to 16,000 feet was decreased from $3^{2}$ to 16 minutes, and that this amount of supercharging apparently did not injure the engine.

The Comparative Performance of Roots' Type Aircraft Engine Superchargers as Affected by Change in Impeller Speed and Displacement. Marsden Ware and E. E. Wilson. N.A.C.A. Report No. 284. Washington, 1928.

\section{Summary.}

Results of tests on three sizes of Roots' type aircraft engine superchargers. From the tests it is evident that impeller speed and displacement have an 
appreciable effect on the performance characteristics of Roots' superchargers aside from their effect as a result of a direct proportional relation. It may be concluded, however, that the speed of impeller operation may be increased to at least 6,000 r.p.m. without imposing any serious performance limitation-the volumetric efficiency is not seriously reduced and the power required per pound of air delivered is not increased excessively at this speed. The results obtained with the 4 -inch supercharger indicate that good performance characteristics may be obtained with this relatively small machine, which lends itself to a compact type of construction so much desired in aircraft practice.

\section{Fluid Motion}

Some Experiments on Motion of Fluids. Part IV. T. Terada and K. Hattori. Report of the A.R.I., Tokyo Imperial University. August, 1927.

\section{Summary.}

From the experimental results it may be seen that the formation of the regularly periodic columnar vortices around a rotating cylinder, as was studied by G. I. Taylor, may be regarded as a special case of a wide class of vortical motions caused by rotation of body in liquid at large.

\section{Fuels-Detonation}

Lower Limit of Inflammability of Ethyl Alcohol, Ethyl Ether, Methyl Cyclohexane and their Mixtures. Y. Tanaka, Y. Nagai and K. Akiyama. Report of the A.R.I., Tokyo Imperial University, No. 2 I. March, 1927.

\section{Summalis.}

The authors describe the results of their research on the lower limits of inflammability of the above. The results showed that the lower limit of inflammability of ethyl alcohol, ethyl ether and methyl cyclohexane for open firing are 3.8I per cent., I.93 per cent. and I.I5 per cent. respectively, and that the theoretical flame propagation temperatures of methyl cyclohexane are nearly the same as those of paraffin hydrocarbons, showing that the naphthene ring has no effect on the theoretical flame propagation temperatures of hydrocarbons. It was also shown that in all mixtures examined, Le Chatelier's rule holds well, the deviation being of much the same order as the experimental error.

\section{Helicopters}

On the Horizontal Flight of a Helicopter. H. Glauert, M.A. R. \& M. No. I, I57 (Ae. 322). H.M.S.O., March, 1928 .

\section{SUMMaRY.}

In a previous report (R. \& M. No. II $3^{2}$ ) a theory has been developed for the vertical ascent of a helicopter. The analysis has now been extended to cover the case of horizontal flight also. The analysis is confined to the case when the helicopter is in horizontal flight with its axis vertical. The effect of variation of the blade angle is considered and also the effect of the periodic motion on the behaviour of airscrews mounted on the blades. The effect of the horizontal motion is to reduce the power required for sustentation. The drag force is not high and the helicopter should therefore be able to attain a satisfactory horizontal. speed. 


\section{Hydrogen}

Studies on the Inflammability of Hydrogen. Parts I.-IV. Y. Tanaka and Y. Nagai. Report of the A.R.I., Tokyo Imperial University. April, 1927May, 1927. No. 22.

\section{Summary.}

The reports deal with the influences of ethyl bromide, organic bromine and iodine compounds, di-ethyl selenide and hydrogen selenide on the limits of inflammability of hydrogen air mixtures.

\section{Instruments}

Report on the Development of a Hot-Wire Rate of Descent Meter. G. W. H. Gardner, B.Sc., and F. W. Meredith, B.A. R. \& M. No. I, I44 (Ae. 3 ${ }^{\mathrm{I} 2}$ ). H.M.S.O., 1928.

\section{Summary.}

The purpose of the investigation was to provide an instrument for aerodynamic research which would indicate varying rates of descent of an aeroplane with an accuracy of \pm Ift. per second.

The instrument developed will indicate the rate of descent of an aeroplane within a range of o-6oft. per second with an accuracy of $\pm \mathrm{Ift}$. per second and has an indication time-lag to $\mathrm{I} / e$ of its amplitude of less than one second. The instrument is bulky and is not recommended for other purposes than aerodynamic research. At the request of the Aeronautical Research Committee a rate of descent recorder involving the principle of the existing hot-wire rate of descent indicator is now being designed.

\section{Instruments-Air Flow}

Measurement of Variable Velocity Relative to Air with Pitot Static Tubes. K. Wada and S. Nisikawa. Report of the A.R.I., Tokyo Imperial University, No. 27. November, 1927.

\section{SUMmary}

Among others the following are discussed in the paper:-(1) Relation between the velocity of a pitot static tube and its pressure indication when the latter was not constant in time. (2) Influence of the friction between the air and the solid wall of the pipe, which connects a pitot static tube to a manometer on the propagation of pressure through it. (3) Relation between the volume capacity of the pressure chamber of a manometer and the pressure indicated.

\section{Materials-General}

Researches on Springs. 3, Torsional Fatigue Tests on Spring Steels. H.M.S.O., 1928.

\section{SUmmary.}

In general, the values of the safe ranges for reversed and repeated stresses are roughly proportional to the tensile strength of the various materials; the ratios of the safe ranges for reversed stresses to the ultimate tensile strengths are about the same as those obtained for normalised steels in the Stromeyer machine.

For the hardened and tempered spring steels the chrome vanadium is slightly superior to the 0.6 per cent. carbon stee], although the tensile strengths are the same. The silico manganese steel, although of greater tensile strength than the chrome vanadium steel, does not give higher safe ranges. Apparently, the values of the safe ranges for repeated stresses are higher than those usually 
obtained from actual springs in service, but the present tests were carried out on carefully prepared specimens in which all surface defects had been reduced to a minimum.

In the case of hardened and tempered spring steels, there are marked decreases in the safe ranges at the high mean stresses, but the results are still considerably above the modified Goodman values.

Stresses in a Plate Bounded by a Hyperbolic Cylinder. A. A. Griffith, D. Eng. R. \& M. No. I. $5^{2}$ (M. 55). H.M.S.O. January, 1928.

\section{Summary.}

The problem is attacked by the method of finding solutions for the case of a plate bounded by a hyperbolic cylinder, which in the limit gives a representation of a solid containing two long cracks which nearly meet. Two solutions are obtained (I) for the case of a shearing load and (2) for the case of a tensile load.

The theory indicates that in a solid containing cracks of the type described, certain elements can undergo elastic rotations of the order of $5^{\circ}$ to $20^{\circ}$. In the case of crystals this inference suggests a possible experimental means of establishing the existence of such cracks or their equivalents.

\section{Materials-Duralumin}

Corrosion Embrittlement of Duralumin. I. Practical aspects of the problem. II. Accelerated corrosion tests and the behaviour of high strength aluminium alloys of different compositions. III. Effect of the previous treatment of sheet material on the susceptibility to this type of corrosion. IV. The use of protective coatings. Henry S. Rawdon. N.A.C.A. Technical Notes Nos. 282-285. Washington, April, 1928.

Summary.

PART I.

Duralumin referred to in these papers is taken as referring to the class of heat-treatable alloys containing $\mathrm{Cu}, \mathrm{Mg}, \mathrm{Mn}$ and $\mathrm{Si}$, and not to the product of any particular manufacturer. It can be positively stated that embrittlement results from sources outside the duralumin and is not spontaneous disintegration of the material itself. The danger from embrittlement decreases as the cross section of the duralumin increases. The purpose of these reports is to develop methods of improvement and protection which would ensure the reliability and permanence of duralumin.

\section{PART II.}

Corrosion of aluminium alloys of the duralumin type shows itself either as a roughening (pitting) of the surface or a penetration into the body of the metal, the path by which it progresses invariably being an intercrystalline one. In the second case the external appearance gives no true indication of the change which has taken place and the breaking load may become very small. The ordinary loss-of-weight corrosion tests are useless in the second case. Experiments were carried out to obtain the intercrystalline effects under conditions representative of those obtaining in aircraft in service. Chloride solutions and halogen solutions caused the intercrystalline effects, but nitrates, sulphates and carbonates had no appreciable corrosive effect and caused no intercrystalline attack.

Of the alloying elements copper appears to be most closely associated with the propensity of the material towards intercrystalline corrosion. The method of heat treatment is important. With certain solutions the corrosion rate is rapidly increased with increase of temperature. 
X-ray examinations by the pin-hole method do not afford a sure means for distinguishing sheet duralumin which is very susceptible to intercrystalline corrosion from that which is not.

A good bibliography is attached to the paper.

PART III.

The results summarised in this report show conclusively that the tendency of sheet duralumin and the related high-strength aluminium alloys to corrode in an intercrystalline manner is intimately related to the method of neat treatment used for the material.

\section{PART IV.}

Protection of the surface is still necessary if long life under varied service conditions is to be ensured. On many points concerning the service behaviour of the coatings, information which can be gained only from the weather exposure tests now under way is necessary before final conclusions can be drawn.

Coatings used may be conveniently grouped into three classes: The varied type of coatings, which are non-metallic in their nature and are applied by brushing, spraying, or dipping; the oxide type, produced by a chemical treatment of the metal whereby the surface metal is converted into a film of oxide or some similar compound; and metallic coatings, of which aluminium appears to be the most promising.

The newer coating methods which appear most promising on the basis of the evidence so far obtainable are $(a)$ metallic aluminium; $(b)$ oil or grease over the anodic coating (with frequent re-oiling for thin oil coats or with aluminium powder over heavy coats); (c) tight-clinging rubber cement carrying, or covered by, aluminium powder-do not readily crack, flake nor bare the duralumin beneath, on weathering and vibration, which is in marked contrast to the ordinary "aluminised" varnish or bitumastic coatings.

\section{Materials, Steel}

The Determination of the Elastic Moduli of a Mild and a Medium Steel. H. E. Smith, B.Sc., and H. L. Cox, B.A. R. \& M. No. I, I 38 (M. 53). H.M.S.O., June, 1927.

\section{Summary.}

The general object of the research was to determine as accurately as possible the elastic contents of certain materials, particularly the standard research materials of the A.R.C. The authors measured $E_{1}, \sigma_{12}$ and $E_{2} ; \sigma_{21}$ was then deduced from the equation $\sigma_{12} / E_{1}=\sigma_{21} / E_{2}$ and $\sigma_{21}$ was also determined directly, and the two values compared. $E_{1}$ and $\sigma_{12}$ were determined from the results of longitudinal and lateral displacement measurements on a circularly cylindrical solid specimen subjected to a simple tensile stress in the direction of the axis of the specimens. $E_{12}$ was determined from the results of lateral displacement measurements on a similar hollow specimen subjected to internal pressure, the end stress due to this pressure being allowed to bear on the specimen. The symbols are:-

$E_{1}=$ Young's modulus in the direction of the $z$ axis.

$E_{2}=$ Young's modulus in the transverse direction.

$\sigma_{12}=$ Ratio of the transverse contraction to the longitudinal extension.

$\sigma_{12}=$ Ratio of the longitudinal contraction to the transverse extension.

All the tests were tensile tests. 


\section{Medical}

Experimental Study on the Effects of Low Barometric Pressures and Oxygen Deprivation upon the Efficiency of Mental and Physical Work. K. Tanaka. Report of the A.R.I., Tokyo Imperial University, No. 37. March, 1928.

\section{Contents.}

The effects of changes of barometric pressures upon the efficiency of mental and physical work; the effects of changes of barometric pressures upon the muscular strength; the effects of changes of barometric pressures upon handwriting; the effects of changes of barometric pressures upon the reaction time; the effects of supplying oxygen.

\section{Meteorology}

Correlations of World Weather and a Formula for Forecasting the Height of the Parana River. E. W. Bliss, M.A., B.Sc., A.Inst.P., F.R.Met.Soc. February, 1928.

\section{Summary.}

Continuing the study of world weather, the following three centres have been added to those previously examined, St. Vincent temperature, S. Rhodesia rainfall, and the height of the Parana river. A formula is obtained on which is based a forecast in September of the probable height of the river in November to May.

Some Studies in Terrestrial Radiation. G. C. Simpson, C.B., F.R.S., F.R.Met.Soc. Memoirs of the Royal Meteorological Society, Vol. II., No. I6. March, 1928.

\section{Stmmary.}

It is assumed that water vapour is the only, constituent of the atmosphere which absorbs and emits long wave radiation. From the results of the upper air observations approximate values of the temperature and water content of the atmosphere at all heights and in all latitudes are adopted. Knowing the amount of water vapour and the temperature, it is possible to calculate the outgoing radiation if the absorption coefficient of water vapour is known. As the outgoing radiation is practically independent of the temperature of the surface, the problem arises as to how the temperature of the atmosphere readjusts itself to changes in solar radiation. This problem is considered in detail, but no solution is found.

Further Studies in Terrestrial Radiation. G. C. Simpson, C.B., F.R.S., F.R. Met. Soc. Memoirs of the Royal Meteorological Society. Vol. III., No. 21. London, July, iga8.

\section{SUMmary.}

By using the observed temperatures of the earth's surface and of the stratosphere, and observed values for the absorption coefficients for water vapour and carbon dioxide, approximate values have been obtained for the outgoing radiation from the earth and its atmosphere, and the laws governing nocturnal radiation have been indicated. Values have been found for the horizontal transfer of heat across the circles of latitude which is necessary to obtain radiative equilibrium of the atmosphere as a whole. The consequences of changes in the intensity of solar radiation have been investigated, and the conclusions drawn that change in cloud amount would be the chief agency by which radiative equilibrium would be restored. An increase in solar radiation would result in increased cloud and 
precipitation, while a decrease in solar energy would lead to less cloud and less precipitation. The possibility of increased solar activity leading to an " ice age " is discussed.

Harmonic Analogies and the Interpretation of the Results of Periodogram Investigations. D. Brunt, M.A., B.Sc., F.R.Met.Soc. Memoirs of the Royal Meteorological Society, Vol. II., No. 15. March, 1928.

\section{Summary.}

The paper collects together for convenience of reference the formulæ used in harmonic analogies.

On Errors in the Multiple Correlation Coefficient Due to Random Sampling. J. Wishart, M.A., B.Sc. Memoirs of the Royal Meteorological Society, Vol. II., No. I3. February, 1928.

\section{SUMmaRY.}

The use of the multiple correlation coefficient in weather forecasting was suggested by Sir Gilbert Walker in I9ı. The author has approached the problem experimentally. The experiment showed, and theory confirmed, that when there is no real multiple correlation between the factors studied, definite values for the mean of a large number of samples, and for the variance, occur. The form of the results for the more general case of real relationship existing between the factors is suggested, but the theory is still far from complete.

On the Mechanism of Cyclones and Anti-Cyclones. Part II. T. Kobayasi. Report of the A.R.I., Tokyo Imperial University, No. 20. March, 1927.

\section{Summairy.}

Gives examples of lines of discontinuity and boundaries of centripetal current drawn on weather charts; lines of discontinuity in anti-cyclones; the translational motion of cyclones and anti-cyclones.

The paper is a continuation of a previous paper with the same title.

\section{Seaplanes}

A Dangerous Seaplane Landing Condition. T. Carroll. N.A.C.A. Technical Note, No. 287. Washington, May, 1928.

- Summary.

A peculiar phenomenon in seaplane landing is observed and reported. The seaplane having executed a normal fast landing at low incidence a forward movement of the control stick effected an unusual condition in that the seaplane left the water suddenly in an abnormal attitude. The observations describing the phenomenon are offered as a warning against possible accident and as a conjectural cause of seaplane landing accidents of a certain kind.

The Reaction on a Float Bottom when Maling Contact with Water at High Speeds. H. C. Richardson. N.A.C.A. Technical Note, No. 288. Washington, May, 1928 .

\section{Stummary.}

Gives an explanation of the phenomenon observed in Technical Note No. 287, and adds: "I would like to emphasise the fact that it may be very dangerous to fly at low angles of attack and high speeds just above the surface of the water. in this position an accidental contact with the surface of the water may lead to a serious crash." 


\section{Wind Tunnels}

The Effect of the Static Pressure Gradient on the Drag of a Body Tested in a Wind Tunnel. H. Glauert, M.A., F.R.Ae.S. R. \& M. No. Ir $5^{8}$ (Ae. 323). H.M.S.O., March, Ig28.

\section{SLMMAI:}

A correction has usually been applied to the observed drag of a body tested in a wind tunnel to allow for the effect of the observed static pressure gradient on the hypothesis that the additional drag experienced by the body is equal to the product of the volume of the body and of the observed pressure gradient.

An attempt is made in this paper to establish the correction on a more rational basis.

The correction is shown to be proportional to the product of the pressure gradient and of a certain effective volume, which is in all the cases examined, greater than the volume of the body, and it is shown that there is such a correction even in the case of a flat plate normal to the wind. The conventional formula is possibly sufficiently accurate in most cases, but for high accuracy, when the correction is required to be in error by less than 20 per cent.; the effective volume should be calculated by the method suggested in the report.

\section{A Theoretical Estimate of the Pressure Gradient in a Wind Tunnel. H. Glauert,} M.A., F.R.Ae.S. $\quad$ R. \& M. No. II59 (Ae. 324). H.M.S.O., April, 1928.

\section{SUMmary.}

The pressure gradient which is known to occur in wind tunnels of the N.P.L. type gives rise to an additional drag force on a model above that which would occur in a uniform stream.

A theoretical expression has been obtained for the pressure gradient due to the development of a boundary layer of retarded air along the walls of a wind tunnel, and as the value so obtained was less than that observed, the analysis has been extended to examine the effect of any leaks in the tunnel walls. A satisfactory explanation of the observed values is then obtained.

The observed pressure gradient depends only in part on the development of the boundary layer, and subsidiary causes, such as leaks in the tunnel walls, may be equally important. It is important, therefore, to avoid any leaks near the working section of a wind tunnel when testing a model whose drag is - susceptible to the pressure gradient.

Variable Density Wind Tunnel. Report of the Scale Effect Panel. R. \& M. No. I, 149 (Ae. 316). H.M.S.O., 1928.

The Panel is satisfied that the variable density wind tunnel has justified its place in the essential apparatus of a first-class aerodynamic laboratory. The provision of such a tunnel would enable a large amount of experimental work to be done in the laboratory which otherwise would only be conducted much more slowly and at a greater cost in free flight. The Panel is of the opinion that the tunnel should not be regarded as a means of reducing the present full scale facilities, but as a means of accelerating and extending aerodynamic research. So far as can be seen, full scale experiments must remain the ultimate standard of reference. It is expected that the value of the variable density tunnel will be found chiefly in effecting a reduction of landing speeds, investigating control at low speeds, and improving performance by the reduction of drag. The Panel strongly recommends the construction of a variable density wind tunnel in this country. 